Research Article

Evolutionary Genetics

\title{
Survey for positively selected coding regions in the genome of the hematophagous tsetse fly Glossina morsitans identifies candidate genes associated with feeding habits and embryonic development
}

\author{
Lucas Freitas $^{1,2,3}$ id , Rafael D. Mesquita ${ }^{2,3}$ iD and Carlos G. Schrago ${ }^{1}$ (iD \\ ${ }^{1}$ Universidade Federal do Rio de Janeiro, Departamento de Genética, Rio de Janeiro, RJ, Brazil. \\ ${ }^{2}$ Universidade Federal do Rio de Janeiro, Instituto de Química, Departamento de Bioquímica, Laboratório \\ de Bioinformática, Rio de Janeiro, RJ, Brazil. \\ ${ }^{3}$ Instituto Nacional de Ciência e Tecnologia em Entomologia Molecular, Rio de Janeiro, RJ, Brazil.
}

\begin{abstract}
Tsetse flies are responsible for the transmission of Trypanossoma sp. to vertebrate animals in Africa causing huge health issues and economic loss. The availability of the genome sequence of Glossina morsitans enabled the discovery of several genes related to medically important phenotypes and novel physiological features. However, a genome-wide scan for coding regions that underwent positive selection is still missing, which is surprising given the evolution of traits associated with the hematophagy in this lineage. In this study, we employed an experimental design that controlled for the rate of false positives and we performed a scan of 3,318 G. morsitans genes. We found 145 genes with significant historical signal of positive selection. These genes were categorized into 18 functional classes after careful manual annotation. Based on their attributed functions, we identified candidate genes related with feeding habits and embryonic development. When our results were contrasted with gene expression data, we confirmed that most genes that underwent adaptive molecular evolution were frequently expressed in organs associated with key physiological evolutionary innovations in the G. morsitans lineage, namely, the salivary gland, the midgut, fat body tissue, and in the spermatophore.
\end{abstract}

Keywords: Tsetse fly, comparative genomics, Diptera, adaptive molecular evolution, neglected tropical disease.

Received: November 3, 2018; Accepted: August 23, 2019.

\section{Introduction}

The Glossinidae consists of an African family of flies known as tsetse, which are the vectors responsible for the transmission of Trypanossoma sp. to humans and other vertebrates. In humans, trypanosomiasis is known as sleeping sickness, whereas a pathologic condition dubbed 'nagana' is reported in other vertebrates (Büscher et al., 2017). These flies are naturally distributed all over the rural areas of sub-Saharan Africa, being endemic to 36 territories, where approximately 70 million people are at risk (Simarro et al., 2012).

In addition to its medical importance, this neglected tropical disease is also responsible for an annual loss of billions of dollars for the livestock industry, as a consequence of diseased farm animals, making animal husbandry extremely hard in infected areas (Kristjanson et al., 1999). Many efforts are being undertaken to overcome trypano-

Send correspondence to Carlos G. Schrago. Universidade Federal do Rio de Janeiro, Instituto de Biologia, Departamento de Genética, CCS, A2-092, Rua Prof. Rodolpho Paulo Rocco, S/N, Cidade Universitária, 21.941-617, Rio de Janeiro, RJ, Brazil. E-mail: carlos.schrago@gmail.com. somiasis. In 2009, for the first time in 50 years, the number of new reports in humans dropped to $<10,000$ cases, and this trend has been kept steady due to WHO efforts in committed areas (WHO, 2017a). The genome of the tsetse fly Glossina morsitans was finished in 2014 (International Glossina Genome Initiative, 2014), and subsequent works identified the function of a number of genes in this species (Benoit et al., 2014a,b, 2015, 2017; Christoffels et al., 2014).

However, although genome sequencing was completed, no genome-wide analysis of positive selection was ever carried out on G. morsitans, in order to gain insights on historical signatures of adaptive molecular evolution. These scans are an essential step in comparative genomics analytical pipelines, and they are possible by the increase in the availability of genomes of closely related species (Ellegren, 2014). These genome-wide searches are also relevant to understand the origin of evolutionary innovations, as positively selected genes (PSG) are likely associated with the emergence of novel phenotypes (Nielsen, 2005), which are frequently associated with adaptive response to new environmental conditions, enabling changes of habit/lifestyle. 
For instance, recent studies have reported positive selection on genes associated with the emergence of eusociality in Hymenoptera (Zhou et al., 2015), the evolution of hypoxia in marine mammals (Foote et al., 2015) and adaptation to carbohydrate diet in humans (Pontremoli et al., 2015).

Two key physiological traits of G. morsitans require major evolutionary transitions, namely, viviparity and the blood-feeding (hematophagous) habit. The former is an unusual pattern of reproduction in Diptera, while the latter arose independently several times in different animals, e.g., insects, annelids, and vertebrates (Azar and Nel, 2012). There are two major compatible hypotheses to explain the evolution of blood-feeding habit in insects: the result of a prolonged association with vertebrates, or a morphological pre-adaptation for piercing (Lehane, 2005). Understanding the evolutionary mechanisms that allowed the evolution of hematophagy in insects is important because most hematophagous species are vectors of several diseases (WHO, 2017b).

It has been proposed that hematophagy evolved independently more than ten times in Insecta (Azar and Nel, 2012), which is indicative of the adaptive value of this feeding habit. Despite its importance, few studies have addressed the evolution of this trait using genomic data (Neafsey et al., 2015; Papa et al., 2017). Furthermore, statistical tests implemented were not able to identify lineage-specific positively selected amino acid sites in hematophagous insects yet, which can be later used in advancing new strategies to control these insect vectors (Anisimova, 2015).

In this sense, G. morsitans represents an excellent case-study, as it exhibits exclusive characteristics, like hematophagy and viviparity, which are not shared with Drosophila melanogaster, its closest sister-lineage with available genome sequence. Therefore, in this study, by performing a comparative evolutionary analysis using an experimental design to control for the rate of false positives, we disclosed genes that evolved under positive selection in the G. morsitans lineage alone.

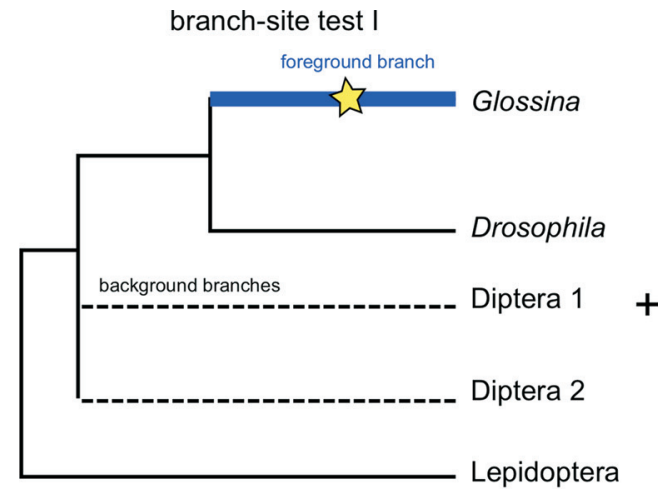

\section{Material and Methods}

\section{Data preparation}

We used publicly available protein coding sequences of seven Diptera and five Lepidoptera species from different databases (Table S1). We focused on 1:1 orthologous coding regions annotated in OrthoDB v.8 (Kriventseva et al., 2014). Each orthologous group was aligned using PAGAN v. 0.56 (Löytynoja et al., 2012) with default parameters. We applied a custom sequence quality filter to remove sequences shorter than half the average sequence length in each orthologous group. Subsequently, we excluded orthologous groups containing fewer than five species. We established that the minimum species sampling consisted of four Diptera species, which were used to increase the power and specificity of our analysis. Tree rooting was accomplished by employing a Lepidoptera species as outgroup (Figure 1). Because we aimed to identify genes related with hematophagy and embryogenesis evolving under positive selection exclusively in G. morsitans, our experimental design included $D$. melanogaster, the sisterlineage in which such traits are absent, in all orthologous groups analyzed. Our final dataset consisted of 3,318 alignments of orthologous coding regions.

\section{Evolutionary analysis and experimental design}

We inferred the gene tree of each orthologous group using RAxML 8.1.17 (Stamatakis, 2014) under the GTR $+\Gamma$ model (Yang, 1996). Standardization of the substitution model was implemented because GTR is the model that best fits most genes (Ranwez et al., 2007), the same rationale was previously employed by Douzery et al. (2014). We used a likelihood ratio test, the Branch-site test 2 (Zhang et al., 2005), as implemented in the codeML program of the PAML 4.8 package (Yang, 2007), to identify amino acid (codon) sites evolving under positive selection in the G. morsitans lineage alone. Positive selection indicates that natural selection favored nucleotide changes lea-

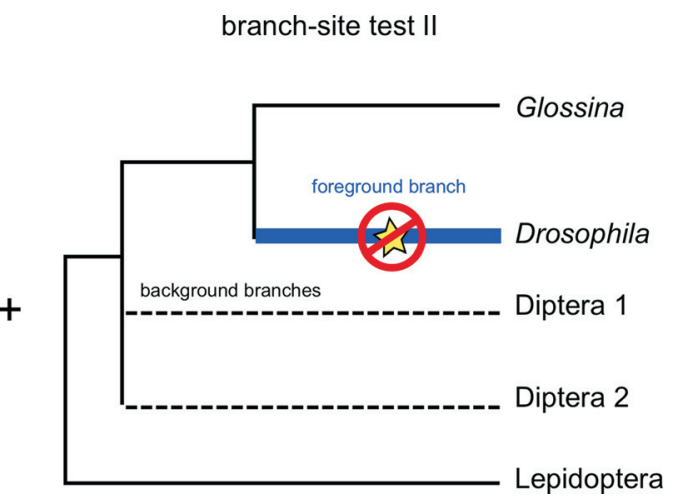

Figure 1 - Two-step test which was used to cross-validate positive selection analysis. In Test I, the branch-site test 2 implemented in PAML was used to identify genes (and their respective codon sites) that underwent positive selection in the branch leading to Glossina (foreground branch). In Test II, the same procedure was implemented in the non-hematophagous Drosophila lineage. We eliminated all genes that were inferred as positively selected in both lineages to gather the set exclusive to Glossina. 
ding to new amino acids (and possibly new functions) in protein sequences; it is inferred when the rate of nonsynonymous substitution $(d N)$ surpasses the synonymous substitution rate $(d S)$, i.e., $d N / \mathrm{dS}>1$ (Yang and Bielawski, 2000).

While conducting the branch-site tests, branches in the phylogeny were divided into foreground and background lineages, where the foreground lineage was allowed to have sites evolving under positive selection. To control for the rate of false positives, we applied a false discovery rate (FDR) approach to multiple testing (Benjamini and Hochberg, 1995). For each of the 3,318 alignments investigated, we performed two independent tests: one assigning the branch leading to G. morsitans as the foreground lineage, and another assigning the branch leading to $D$. melanogaster as the foreground. Therefore, we conducted a total of 6,636 tests in which the FDR correction was applied. A positively selected gene was only deemed as exclusive to the G. morsitans lineage if no positive selected codon site was inferred in that same alignment when $D$. melanogaster was used as a foreground lineage (Figure 1). Therefore, if a given coding region was inferred to have undergone positive selection in Tests I and II on different codon sites, it was also discarded. This two-step experimental design was adopted as a methodological cross-validation approach to avoid false positives.

Positively selected codon sites were inferred according to the Bayes empirical Bayes (BEB) approach developed by Yang (2005). If the BEB approach inferred a gene with at least one site with posterior probability $>95 \%$ of belonging to the $d N / d S>1$ class, we considered that this gene evolved under positive selection. Because of the large extent of the divergence times between species studied (Misof et al., 2014), we expect the presence of a high degree of saturation of synonymous sites that may lead to errors in the alignments. This issue will impact the quality of the alignment, but not the inference of positive selection (Fletcher and Yang, 2009). Hence, we included another precautionary step, by manually excluding genes in which the positively selected site was located in low-quality alignment regions. We also excluded positively selected codon sites that coded for serine, because such substitutions may incorrectly be identified to be under positive selection, increasing the rate of false-positives (Yang and dos Reis, 2011). Due to the several filters applied, we expect our analytical procedure to be conservative, reducing the type I error rate. Thus, we preferred a statistical framework in which the type II error rate might have increased, instead of providing over credible evidence of molecular adaptation.

\section{Manual functional annotation and comparison with expression data}

While surveying gene functions related with hematophagy and embryonic development, we performed extra checks of the functions of annotated coding regions relying on multiple databases: FlyBase (Gramates et al., 2017), PantherDB (Mi et al., 2017), PFAM (Finn et al., 2016), InterPro (Finn et al., 2017), UniProt (The UniProt Consortium, 2016) and NCBI databases (NCBI Resource Coordinators, 2017). Additionally, we used gene expression data from several works, performed on different tissues (Lehane et al., 2003; Attardo et al., 2006; Alves-Silva et al., 2010; Telleria et al., 2014; Scolari et al., 2016; Bing et al., 2017), to verify the presence of the estimated PSGs. In some works, the list of expressed genes was not available with the proper VectorBase gene ID (Lehane et al., 2003; Attardo et al., 2006; Alves-Silva et al., 2010). We therefore used the IDs available in the GM-s1-Web.xls table of (Alves-Silva et al., 2010) to download all these sequences and then, performed a BLAST v. 2.2.31 (Altschul, 1990) search against the new annotation. The correspondence between both IDs was established and the information available on gene expression was used to detect the presence of PSGs in each tissue-specific expression data.

\section{Results}

Using the branch-site test, we were able to identify 145 genes with at least one codon site evolving under positive selection exclusively in G. morsitans (Table S2). The manual functional annotation of these PSGs categorized 140 genes into 18 categories (Figure 2, Table S2). The largely recovered categories were transcription/translation (32), folding/protein degradation (16), cell signaling (15), development (12) and replication/DNA maintenance (12).

After annotation, 12 genes were identified as related with embryonic development (Table S2), as they were attributed to the class development. Regarding coding regions putatively associated with hematophagy, we identified six genes as potential candidates related with this complex trait due to their role on amino acid metabolism and stress response. Genes GMOY005584 and GMOY010949 participate in alanine-glycine transamination and tryptophan degradation respectively; whereas GMOY004385 is an amino acid transmembrane transport protein. GMOY002159 and GMOY004064 are

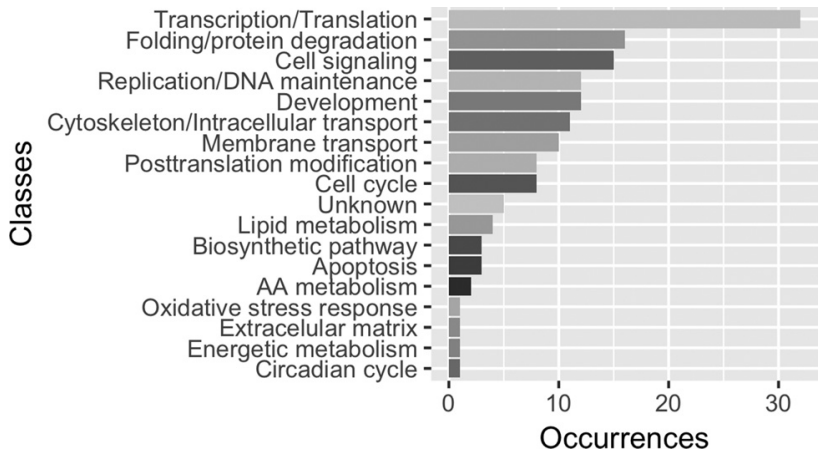

Figure 2 - Distribution of positively selected genes exclusive to Glossina morsitans according to their functional class. 
involved in DNA repair, and GMOY009602 acts on the oxidative stress response. One gene, GMOY005584, is worthy of attention due to its dependence of vitamin B6 produced by the endosymbiont Wigglesworthia glossinidia (Michalkova et al., 2014).

Furthermore, two major cellular processes stood out among PSGs, cell maintenance and DNA-mRNA-proteinfunction balance. Annotation classes (Table S2) included in the cellular processes were: replication/DNA maintenance (12 genes), apoptosis (3), and cell cycle (8); while those associated with protein function were: transcription/translation (32, of which 7 are transcription factors), post-translational modification (8), and protein folding degradation (16).

After comparison with tissue-specific gene expression data, most coding regions were inferred to have undergone adaptive evolution: 109 out of 145 were expressed in organs associated with key evolutionary innovations (Table S3), from which 18 were exclusively found in the salivary glands, 16 in the midgut, 12 in the fat body, and two genes (GMOY011979 and GMOY005584) in the spermatophore.

\section{Discussion}

We were able to identify a total of 145 genes evolving under positive selection in the G. morsitans lineage, and our manual annotation identified several candidate genes related to embryonic development and feeding habit. Furthermore, functional classes that were associated with these phenotypes were well represented among PSGs. For instance, genes assigned to protein folding/degradation (16 genes), replication/DNA maintenance (12), cell cycle (8), and apoptosis (2) categories, presumptively deal with the oxidative stress caused by the free heme group during blood-digestion (Sterkel et al., 2017).

Our experimental design was structured in order to control for false positives that may arise when comparing sequences separated by several million years. Adaptive molecular evolution is ultimately inferred by comparing non-synonymous and synonymous distances. Because synonymous sites evolve faster than non-synonymous sites, saturation of evolutionary distance is more likely to impact synonymous changes, decreasing the accuracy of the branch-site test (Gharib and Robinson-Rechavi, 2013). To reduce this effect, we conducted a cross-validation hypothesis testing, applying false discovery rate and carefully filtered gap-rich regions in the alignment. As a result, we expect that the type I error was undermined, despite the evolutionary distances between the species used in our dataset.

The adaptive mutations that gave rise to hematophagy in the G. morsitans lineage were fixed in the ancestor of the lineage. Therefore, although a single Calyptratae genome (G. morsitans) was investigated, we expect that, when compared to Drosophila, substitutions associated with the evolution of these complex traits were correctly inferred along the branch leading to G. morsitans. Evidently, in order to single out those substitutions exclusively associated with the emergence of hematophagy, further experimental analyses are required. Especially, the analysis of non-hematophagous species that are evolutionarily closer to Glossina should bring further statistical sensitivity to the inference of PSGs associated with this complex trait. We argue, however, that contrasting our results against gene expression data from key tissues is a promising first step in order to gather a set of candidate genes by bioinformatics.

The expression of PSGs in the four tissues where high-throughput data was available (Lehane et al., 2003; Attardo et al., 2006; Alves-Silva et al., 2010; Telleria et al., 2014; Scolari et al., 2016) indicated that 66\% (96 out of 145 PSGs) were expressed in the salivary glands and/or midgut tissues. We argue that this provides further corroboration and insights on the putative association of our set of PSGs with hematophagy, as these tissues are clearly involved in the feeding success by their hemostasis counteraction (Ribeiro et al., 2010) and blood digestion.

The salivary gland (Alves-Silva et al., 2010; Telleria et al., 2014) was the organ with the largest number of PSGs (82 out of 145). Previous studies have reported that, in insects, salivary gland genes are under strong selective pressure (Arcà et al., 2014; Neafsey et al., 2015). Notwithstanding, most of those salivary gland PSGs are species-specific, and originated by gene duplications (Ribeiro and Francischetti, 2003; Ribeiro et al., 2010). The amino acid replacements in the 82 PSGs estimated here likely consist of evolutionary innovations against hemostasis, and reinforce that the selective pressure for the effectiveness of the saliva proteins is indeed high in blood-feeding insects.

Recently, Bing et al. (2017) analyzed the G. morsitans midgut transcriptome. This organ harbors the obligate endosymbiont $W$. glossinidia, which is required for $G$. morsitans reproduction. Midgut transcriptomes were sequenced under three different conditions: the control (with W. glossinidia), aposymbiotic,, and infected (with Trypanosoma brucei rhodesiense). Compared with the control, five PSGs found here were up-regulated (GMOY000234, GMOY003765, GMOY007531, GMOY008484 and GMOY011346), while one gene was down-regulated (GMOY004385) in both aposymbiotic and infected flies (Table S4). The PSG GMOY009189 was down-regulated only in aposymbiotic flies (Table S4). When control and aposymbiotic flies were compared, 18 PSGs were not differentially expressed, while nine were underexpressed and seven were overexpressed in aposymbiotic flies (Table S4). Among these nine underexpressed genes, GMOY005584 may be a good candidate for understanding the evolution of host-microbiome interaction, as it uses the pyridoxal 5'phosphate (vitamin B6) produced by its endosymbiont as a cofactor (Michalkova et al., 2014).

It is worth mentioning that the positive selection analysis carried out here was restricted to single copy orthologs. 
The evolution of a complex trait, such as hematophagy, may be related with evolutionary novelties not directly linked to substitutions in 1:1 orthologs. For instance, the expansion/contraction of gene families, as well as the neofunctionalization of genes, have also been linked to the emergence of adaptive traits (Assis and Bachtrog, 2013; Seppey et al., 2019). Further analyses are required to assess the role of those alternative modes of genome evolution played in the evolution of hematophagy. Such investigation can be implemented using the same experimental design proposed here to control for false positives.

For drawing a comprehensive scenario of the molecular changes that allowed Glossina flies to feed on blood, additional comparative genomic data is required. Genome sequencing of non-hematophagous lineages closely related to Glossina will increase the accuracy of evolutionary analyses. Moreover, transcriptomes contrasting gene expression under different feeding regimes (blood vs. unfed/sugar) in the salivary gland and/or midgut are needed to further investigate the functional role of PSGs in the blood-feeding habit. Also, gene expression profiles from different embryonic stages of development are desirable. The availability of those comparative data will clarify which genes were associated with the rise of physiological innovations in the G. morsitans lineage, potentially helping with the development new vector control strategies (Chen et al., 2004; Zhang et al., 2013; Anisimova, 2015).

\section{Acknowledgments}

This work is part of the Doctoral thesis of LF funded by the Brazilian Ministry of Education (CAPES- Brasil, Finance code 001) and the Brazilian Research Council (CNPq). CGS was funded by the $\mathrm{CNPq}$ grants 440954/2016-9, 421392/2016-9, 310974/2015-1 and 200332/2018-0. We thank Dr. Thiago Venâncio (UENF) for performing the transcription factor analysis.

\section{Conflict of Interest}

The authors declare no conflict of interest.

\section{Author Contributions}

LF and CGS conceived the study; LF, RDM and CGS designed the experiments; LF and RDM carried out the analysis; LF, RDM and CGS interpreted the results; LF and CGS wrote the manuscript, and all authors approved the final version.

\section{References}

Altschul S (1990) Basic local alignment search tool. J Mol Biol 215:403-410.

Alves-Silva J, Ribeiro JM, Van Den Abbeele J, Attardo G, Hao Z, Haines LR, Soares MB, Berriman M, Aksoy S and Lehane MJ (2010) An insight into the sialome of Glossina morsitans morsitans. BMC Genomics 11:213.
Anisimova M (2015) Darwin and Fisher meet at biotech: On the potential of computational molecular evolution in industry. BMC Evol Biol 15:76.

Arcà B, Struchiner CJ, Pham VM, Sferra G, Lombardo F, Pombi M and Ribeiro JMC (2014) Positive selection drives accelerated evolution of mosquito salivary genes associated with blood-feeding. Insect Mol Biol 23:122-131.

Assis R and Bachtrog D (2013) Neofunctionalization of young duplicate genes in Drosophila. Proc Natl Acad Sci U S A 110:17409-17414.

Attardo GM, Strickler-Dinglasan P, Perkin SAH, Caler E, Bonaldo MF, Soares MB, El-Sayeed N and Aksoy S (2006) Analysis of fat body transcriptome from the adult tsetse fly, Glossina morsitans morsitans. Insect Mol Biol 15:411-424.

Azar D and Nel A (2012) Evolution of hematophagy in "nonbiting midges" (Diptera: Chironomidae). Terr Arthropod Rev 5:15-34.

Benjamini Y and Hochberg Y (1995) Controlling the false discovery rate: a practical and powerful approach to multiple testing. J R Stat Soc Series B Stat Methodol 57:289-300.

Benoit JB, Attardo GM, Michalkova V, Krause TB, Bohova J, Zhang Q, Baumann AA, Mireji PO, Takác P, Denlinger DL et al. (2014a) A novel highly divergent protein family identified from a viviparous insect by RNA-seq analysis: a potential target for tsetse fly-specific abortifacients. PLoS Genet 10:e1003874.

Benoit JB, Hansen IA, Attardo GM, Michalková V, Mireji PO, Bargul JL, Drake LL, Masiga DK and Aksoy S (2014b) Aquaporins are critical for provision of water during lactation and intrauterine progeny hydration to maintain tsetse fly reproductive success. PLoS Negl Trop Dis 8:e2517.

Benoit JB, Attardo GM, Baumann AA, Michalkova V and Aksoy S (2015) Adenotrophic viviparity in tsetse flies: Potential for population control and as an insect model for lactation. Annu Rev Entomol 60:351-371.

Benoit JB, Vigneron A, Broderick NA, Wu Y, Sun JS, Carlson JR, Aksoy S and Weiss BL (2017) Symbiont-induced odorant binding proteins mediate insect host hematopoiesis. Elife 6:e19535.

Bing X, Attardo GM, Vigneron A, Aksoy E, Scolari F, Malacrida A, Weiss BL and Aksoy S (2017) Unravelling the relationship between the tsetse fly and its obligate symbiont Wigglesworthia: transcriptomic and metabolomic landscapes reveal highly integrated physiological networks. Proc Biol Sci 284:20170360.

Büscher P, Cecchi G, Jamonneau V and Priotto G (2017) Human African trypanosomiasis. Lancet 375:148-159.

Chen L, Perlina A and Lee CJ (2004) Positive selection detection in 40,000 human immunodeficiency virus (HIV) type 1 sequences automatically identifies drug resistance and positive fitness mutations in HIV protease and reverse transcriptase. J Virol 78:3722-3732.

Christoffels A, Masiga D, Berriman M, Lehane M, Touré Y and Aksoy S (2014) International Glossina genome initiative 2004-2014: A driver for post-genomic era research on the African continent. PLoS Negl Trop Dis 8:e3024.

Douzery EJP, Scornavacca C, Romiguier J, Belkhir K, Galtier N, Delsuc F and Ranwez V (2014) OrthoMaM v8: A database of orthologous exons and coding sequences for comparative genomics in mammals. Mol Biol Evol 31:1923-1928. 
Ellegren H (2014) Genome sequencing and population genomics in non-model organisms. Trends Ecol Evol 29:51-63.

Finn RD, Coggill P, Eberhardt RY, Eddy SR, Mistry J, Mitchell AL, Potter SC, Punta M, Qureshi M, Sangrador-Vegas A et al. (2016) The Pfam protein families database: Towards a more sustainable future. Nucleic Acids Res 44:d279-285.

Finn RD, Attwood TK, Babbitt PC, Bateman A, Bork P, Bridge AJ, Chang HY, Dosztányi Z, El-Gebali S, Fraser M et al. (2017) InterPro in 2017-beyond protein family and domain annotations. Nucleic Acids Res 45:190-199.

Fletcher W and Yang Z (2009) INDELible: A flexible simulator of biological sequence evolution. Mol Biol Evol 26:1879-1888.

Foote AD, Liu Y, Thomas GWC, Vinar T, Alföldi J, Deng J, Dugan S, van Elk CE, Hunter ME, Joshi V et al. (2015) Convergent evolution of the genomes of marine mammals. Nat Genet 47:272-275.

Gharib WH and Robinson-Rechavi M (2013) The branch-site test of positive selection is surprisingly robust but lacks power under synonymous substitution saturation and variation in GC. Mol Biol Evol 30:1675-1686.

Gramates LS, Marygold SJ, Santos GD, Urbano JM, Antonazzo G, Matthews BB, Rey AJ, Tabone CJ, Crosby MA, Emmert DB et al. (2017) FlyBase at 25: looking to the future. Nucleic Acids Res 45:D663-D671.

International Glossina Genome Initiative (2014) Genome sequence of the tsetse fly (Glossina morsitans): Vector of African trypanosomiasis. Science 344:380-386.

Kristjanson PM, Swallow BM, Rowlands GJ, Kruska RL and de Leeuw PN (1999) Measuring the costs of African animal trypanosomosis, the potential benefits of control and returns to research. Agric Syst 59:79-98.

Kriventseva EV, Tegenfeldt F, Petty TJ, Waterhouse RM, Simão FA, Pozdnyakov IA, Ioannidis P and Zdobnov EM (2014) OrthoDB v8: Update of the hierarchical catalog of orthologs and the underlying free software. Nucleic Acids Res 43:D250-D256.

Lehane MJ (2005) The biology of blood-sucking in insects. 2nd edition. Cambridge University Press, New York, 321 p.

Lehane MJ, Aksoy S, Gibson W, Kerhornou A, Berriman M, Hamilton J, Soares MB, Bonaldo MF, Lehane S and Hall N (2003) Adult midgut expressed sequence tags from the tsetse fly Glossina morsitans morsitans and expression analysis of putative immune response genes. Genome Biol 4:R63.

Löytynoja A, Vilella AJ and Goldman N (2012) Accurate extension of multiple sequence alignments using a phylogenyaware graph algorithm. Bioinformatics 28:1684-1691.

Michalkova V, Benoit JB, Weiss BL, Attardo GM and Aksoy S (2014) Vitamin B6 generated by obligate symbionts is critical for maintaining proline homeostasis and fecundity in tsetse flies. Appl Environ Microbiol 80:5844-5853.

Mi H, Huang X, Muruganujan A, Tang H, Mills C, Kang D and Thomas PD (2017) PANTHER version 11: Expanded annotation data from Gene Ontology and Reactome pathways, and data analysis tool enhancements. Nucleic Acids Res 45:D183-D189.

Misof B, Liu S, Meusemann K, Peters RS, Donath A, Mayer C, Frandsen PB, Ware J, Flouri T, Beutel RG et al. (2014) Phylogenomics resolves the timing and pattern of insect evolution. Science 346:763-767.
NCBI Resource Coordinators (2017) Database resources of the National Center for Biotechnology Information. Nucleic Acids Res 46:8-13.

Neafsey DE, Waterhouse RM, Abai MR, Aganezov SS, Alekseyev MA, Allen JE, Amon J, Arcà B, Arensburger P, Artemov G et al. (2015) Highly evolvable malaria vectors: The genomes of 16 Anopheles mosquitoes. Science 347:1258522.

Nielsen R (2005) Molecular signatures of natural selection. Annu Rev Genet 39:197-218.

Papa F, Windbichler N, Waterhouse RM, Cagnetti A, D'Amato R, Persampieri T, Lawniczak MKN, Nolan T and Papathanos PA (2017) Rapid evolution of female-biased genes among four species of Anopheles malaria mosquitoes. Genome Res 27:1536-1548

Pontremoli C, Mozzi A, Forni D, Cagliani R, Pozzoli U, Menozzi G, Vertemara J, Bresolin N, Clerici M and Sironi M (2015) Natural selection at the brush-border: Adaptations to carbohydrate diets in humans and other mammals. Genome Biol Evol 7:2569-2584.

Ranwez V, Delsuc F, Ranwez S, Belkhir K, Tilak MK and Douzery EJ (2007) OrthoMaM: A database of orthologous genomic markers for placental mammal phylogenetics. BMC Evol Biol 7:241.

Ribeiro JMC and Francischetti IMB (2003) Role of arthropod saliva in blood feeding: Sialome and post-sialome perspectives. Annu Rev Entomol 48:73-88.

Ribeiro JMC, Mans BJ and Arcà B (2010) An insight into the sialome of blood-feeding Nematocera. Insect Biochem Mol Biol 40:767-784.

Scolari F, Benoit JB, Michalkova V, Aksoy E, Takac P, Abd-Alla AMM, Malacrida AR, Aksoy S and Attardo GM (2016) The spermatophore in Glossina morsitans morsitans: Insights into male contributions to reproduction. Sci Rep 6:20334.

Seppey M, Ioannidis P, Emerson BC, Pitteloud C, RobinsonRechavi M, Roux J, Escalona HE, McKenna DD, Misof B, Shin $\mathrm{S}$ et al. (2019) Genomic signatures accompanying the dietary shift to phytophagy in polyphagan beetles. Genome Biol 20:98.

Simarro PP, Cecchi G, Franco JR, Paone M, Diarra A, RuizPostigo JA, Fèvre EM, Mattioli RC and Jannin JG (2012) Estimating and mapping the population at risk of sleeping sickness. PLoS Negl Trop Dis 6:e1859.

Stamatakis A (2014) RAxML version 8: A tool for phylogenetic analysis and post-analysis of large phylogenies. Bioinformatics 30:1312-1313.

Sterkel M, Oliveira JH, Bottino-Rojas V, Paiva-Silva GO and Oliveira PL (2017) The dose makes the poison: nutritional overload determines the life traits of blood-feeding arthropods. Trends Parasitol 33:633-644.

Telleria EL, Benoit JB, Zhao X, Savage AF, Regmi S, Alves e Silva TL, O'Neill M and Aksoy S (2014) Insights into the trypanosome-host interactions revealed through transcriptomic analysis of parasitized tsetse fly salivary glands. PLoS Negl Trop Dis 8:e2649.

The UniProt Consortium (2016) UniProt: The universal protein knowledgebase. Nucleic Acids Res 45:158-169.

Yang Z (1996) Among-site rate variation and its impact on phylogenetic analyses. Trends Ecol Evol 11:367-372.

Yang Z (2005) Bayes empirical bayes inference of amino acid sites under positive selection. Mol Biol Evol 22:1107-1118. 
Yang Z (2007) PAML 4: Phylogenetic analysis by maximum likelihood. Mol Biol Evol 24:1586-1591.

Yang Z and Bielawski JP (2000) Statistical methods for detecting molecular adaptation. Trends Ecol Evol 15:496-503.

Yang Z and dos Reis M (2011) Statistical properties of the branch-site test of positive selection. Mol Biol Evol 28:1217-1228.

Zhang J, Nielsen R and Yang Z (2005) Evaluation of an improved branch-site likelihood method for detecting positive selection at the molecular level. Mol Biol Evol 22:2472-2479.

Zhang G, Cowled C, Shi Z, Huang Z, Bishop-Lilly KA, Fang X, Wynne JW, Xiong Z, Baker ML, Zhao W et al. (2013) Comparative analysis of bat genomes provides insight into the evolution of flight and immunity. Science 339:456-460.

Zhou X, Rokas A, Berger SL, Liebig J, Ray A and Zwiebel LJ (2015) Chemoreceptor evolution in Hymenoptera and its implications for the evolution of eusociality. Genome Biol Evol 7:2407-2416.

\section{Internet resources}

WHO (2017a) Trypanosomiasis, human African (sleeping sickness), http://www.who.int/mediacentre/factsheets/fs259/en/ (accessed 21 November 2017).

WHO (2017b) Vector-borne diseases, http://www.who.int/mediacentre/factsheets/fs387/en/ (accessed 21 November 2017).

\section{Supplementary material}

The following online material is available for this article: Table S1 - Species used in this study and the source of the genomes.

Table S2 - Manual annotation of the 145 positively selected genes.

Table S3 - Distribution of positively selected genes in tissues.

Table S4 - Positively selected genes differentially expressed between control and aposymbiotic/Trypanosome infected fly midguts.

Associate Editor: Louis Bernard Klaczko

License information: This is an open-access article distributed under the terms of the Creative Commons Attribution License (type CC-BY), which permits unrestricted use, distribution and reproduction in any medium, provided the original article is properly cited. 